\title{
Coagulopathy After Viper Snakebite in Vietnam and Relationship with Time of Admission
}

\author{
Xuan Thi Dang (D) ${ }^{1,2}$ \\ Thanh Xuan Nguyen (D) ${ }^{3-5}$ \\ Thu Thi Hoai Nguyen (iD) 3,4 \\ Hung Tran $\mathrm{Ha}^{1,2}$ \\ 'Vietnam Poison Control Center, Bach \\ Mai Hospital, Hanoi, 100000, Vietnam; \\ ${ }^{2}$ Department of Emergency and Critical \\ Care Medicine, Hanoi Medical University, \\ Hanoi, 100000, Vietnam; ${ }^{3}$ Geriatrics \\ Department, Hanoi Medical University, \\ Hanoi, I00000, Vietnam; ${ }^{4}$ Scientific \\ Research Department, National Geriatric \\ Hospital, Hanoi, 100000, Vietnam; ${ }^{5}$ Dinh \\ Tien Hoang Institute of Medicine, Hanoi, \\ I00000, Vietnam
}

Background: Snakebite envenoming is a potentially life-threatening condition and causes many serious consequences.

Subjects and Methods: Therefore, this study aimed to throw some light on coagulopathy after Viperidae envenomations at Vietnam Poison Control Center and the relationship between coagulopathy and time of admission. A prospective, descriptive study was conducted from October 2016 to April 2018. The survey questionnaire included socio-economic characteristics, characteristics of snakebite, signs and clinical symptoms and blood test. Disseminated intravascular coagulation (DIC) condition was diagnosed using the International Society on Thrombosis and Haemostasis (ISTH) criteria. Rotational thromboelastometry was evaluated using ROTEM $^{\circledR}$ delta system.

Results: A total of 41 cases of viper snakebite with the mean age of snakebite victims were $41.27 \pm 14.72$ years old. Mean hospital stay of the patients was $5.63 \pm 3.29$ days. The association between coagulation disorder and clotting time (CT) EXTEM prolonged, CT INTEM prolonged, CT FIBTEM prolonged remained significant (multivariable odds ratio $\mathrm{MOR}=5.81,95 \% \mathrm{CI}: 1.20-28.06 ; \mathrm{MOR}=9.32,95 \% \mathrm{CI}: 1.001-84.48 ; \mathrm{MOR}=5.55,95 \% \mathrm{CI}$ : $1.12-27.50$, respectively).

Conclusion: This study indicates a hypocoagulation status in ROTEM, elevated international normalised ratio (INR), activated partial thromboplastin time (APTT) and D-dimer, decreased fibrinogen concentration and platelet count following envenoming by Viperidae.

Keywords: viper, snakebite, Vietnam, coagulopathy, ROTEM

\section{Introduction}

Snakebite envenoming is a potentially life-threatening condition and causes many serious consequences. Available data showed that each year up to 138000 deaths worldwide due to venomous snakes and as many as 500,000 cases of venominduced morbidity. ${ }^{1}$ South and Southeast Asia have the highest incidence and mortality rates of snakebite in the world, and Vietnam has a high estimated number of envenomings every year $(30,000)^{2}$ with a high mortality rate per year (80 people per million). ${ }^{3}$ The actual incidence of snakebites may be much higher because based on interviews with clinicians, inhabitants and community leaders a rough estimate was that less than $20 \%$ of the snakebite victims visited a clinic. ${ }^{4}$

In Vietnam, 60 venomous snakes have been recorded, comprising 37 species (15 genera) of the family Elapidae and 23 species ( 9 genera) of the family Viperidae. The green pit viper is mostly responsible for number of bites followed by the Malay and Cobra pit viper in Vietnam. ${ }^{5}$ Viper venom composition is characterized by many different toxin families, like cysteine-rich secretory proteins, snake venom
Correspondence: Xuan Thi Dang Vietnam Poison Control Center, Bach Mai Hospital, Hanoi, I00000, Vietnam Tel +84912260366

Email xuandangthi@bachmai.edu.vn 
serine proteases, phospholipases A2, snake venom metalloproteases, C-type lectins, coagulation inhibitors and disintegrins, haemorrhagic factors, which result in venominduced consumption coagulopathy. Viper snake venom affects the coagulation process (pre-coagulation, anticoagulation, thrombin inhibition, fibrinogen resolution, impact on vessel walls, platelets). ${ }^{6-8}$ Clinical manifestations encompass a wide variety of problems including mild symptoms to haemostatic abnormalities, cardiotoxicity, nephrotoxicity, shock and death. ${ }^{6}$

Blood coagulation tests are cornerstone for diagnosis and treatment of snakebite coagulopathy. Understanding of the coagulation effects arising from human envenoming will improve prognosis, management, treatment with antivenom and the role of adjuvant therapies. There are very few studies related to coagulation parameter abnormalities after Viper snakebite in Vietnam. Therefore, we have undertaken this study to throw some light on coagulopathy after Viper snake envenomations at Vietnam Poison Control Center and the relationship between coagulopathy and time of admission.

\section{Materials and Methods}

\section{Study Setting and Participants}

A prospective, descriptive study was conducted at Vietnam Poison Control Center, Bach Mai Hospital, Hanoi, Vietnam. This institute is a referral government hospital, the only Poison Control Center (PCC) in the North of Vietnam. The records of all Viperidae's snakebite victims who attended this center from October 2016 to April 2018 were recruited.

Patients were included if they had a definite history of snakebite and developed features of envenomation. The Viperidae's snakebite was determined when patients had criteria 4 and one of criteria 1,2,3: (1) the presence of fang marks; (2) presence of swelling, ecchymosis, cellulitis, necrosis, blister formation and/or bleeding from local site; (3) systemic symptoms: disturbances in coagulation mechanism with or without systemic bleeding; (4) identification of snake: patient or people involved brings dead snake with or identifies the snake through sample image. Dead snake or photographs were sent to the identification specialist at the Museum of Nature, Vietnam Academy of Science and Technology then confirmed they belong the Viperidae.

Patients were excluded from the study if they had at least one of the following criteria: (1) lack of signs or symptoms of envenomation after a period of observation; (2) with pre-existing renal disease, liver dysfunction and bleeding disorder; (3) have been using anticoagulants; (4) refused to participate in the study.

A total number of 41 Viperidae's snakebite cases during the study period were enrolled in this study.

\section{Measures and Instruments}

After obtaining consent, data was collected on predesigned and structured questionnaire by interviewing patients and their families. The survey questionnaire included the following:

General demographic characteristics: age, gender, occupation

Characteristics of snakebite: place of bite, site of bite, type of snake, time interval to reach the health facility after snakebite; clinical symptoms (bleeding, swelling, necrosis, blister formation from local site, gastrointestinal hemorrhage).

Other signs and clinical symptoms: level of consciousness (using the Glasgow Coma Scale (GCS)), blood pressure, $\mathrm{SpO} 2$ on admission, respiratory rate, heart rate, duration of hospital stay, and clinical outcome (fatal/survival).

Blood tests: blood sample of patients on admission were analysed for complete blood counts (platelet count, hemoglobin), creatinine level and coagulation studies including prothrombin time \% activity (PT \%), international normalized ratio (INR), activated partial thromboplastin time (APTT), thrombin time (TT), serum fibrinogen level, D-dimer level, euglobulin clot lysis time (Von-kaulla test).

Disseminated intravascular coagulation (DIC) was diagnosed using the International Society on Thrombosis and Haemostasis (ISTH) criteria. The criteria include four factors: platelet count, PT \%, fibrinogen level, elevated levels of a fibrin-related marker (D-dimer level). Total score ranges from 0 to 8 points. Total score greater than or equal to 5 suggested overt DIC, score less than 5 not suggesting overt DIC, may be no DIC. ${ }^{7}$

Rotational thromboelastometry (ROTEM) on admission was evaluated using ROTEM $^{\circledR}$ delta system (TEM Innovations $\mathrm{GmbH}$ - Germany). Its variables were clotting time (CT), maximum clot firmness (MCF). The applicability of the variables described was done with the three available tests for real-time analysis of $\operatorname{ROTEM}^{\circledR}$ : INTEM, EXTEM, FIBTEM. ${ }^{8}$

\section{Data Analysis}

All the data obtained were recorded and analyzed using the Statistical Package for the Social Sciences (SPSS), 
version 22.0. Descriptive statistics for participant characteristics were presented as mean $\pm \mathrm{SD}$ for continuous variables and frequency/percentages for categorical variables, respectively. Comparisons between participants with no-DIC, non-overt DIC and overt DIC were assessed using chi-squared test for categorical variables and Student's $t$-tests for continuous variables. Two-tailed $\mathrm{p}$ values $<0.05$ were considered statistically significant.

Multivariate logistic regression was applied to identify associated factors for coagulation disorders (CT EXTEM prolong, CT INTEM prolong, CT FIBTEM prolong, MCF FIBTEM decreased). A p-value of 0.2 was employed as a threshold of variable selection. An adjusted odds ratio (OR) was presented along with the p-value and a 95\% confidence interval. A p-value of less than 0.05 was recognized as statistically significant.

\section{Ethics Approval and Informed Consent}

The study was approved by the Ethics Committee of Bach Mai Hospital. Written in-formed consent was obtained from all participants. For patients who were not of legal age to sign on their own behalf, written informed consent was obtained from their guardians.

\section{Results}

A total of 41 cases of viper snakebite were included in this study. The mean age of snakebite victims was $41.27 \pm$ 14.72 years old. Most of the study patients were males (61\%) and the male to female ratio was $1.56 / 1$. Farmers accounted for nearly half of the victims (46.3\%). Mean hospital stay of the patients was $5.63 \pm 3.29$ days. About $26.8 \%$ of patients reached the hospital after 6 hours of snakebite. Mortality rate was $0 \%$ (Table 1 ).

Comparison of patients according to the presence and severity of DIC using the ISTH scoring system in 41 patients: the normal group comprised 10 patients (24.4\%), the simple coagulopathy group comprised 17 patients $(41.5 \%)$, and the overt DIC group comprised 14 patients (34.1\%). In statistical analysis, snake type, gastrointestinal hemorrhage, and heart rate reached statistical significance. DIC disorder has the highest prevalence in Trimeresurus albolabris snake group. From the results, gastrointestinal hemorrhage and heart rate were significantly more increased in the over DIC group than in the simple coagulopathy and normal group $(\mathrm{p}<0.05)$ (Table 2$)$.

Out of 41 patients, platelet count was decreased in 14 (34.2\%). Prothrombin levels were decreased in 16 (39\%) cases and APTT was prolonged in 7 (17.1\%) cases. INR
Table I General Characteristics $(n=4 I)$

\begin{tabular}{|l|l|l|}
\hline Characteristics & Number & Percentage \\
\hline Male & 25 & 61 \\
\hline Occupational & \multicolumn{2}{|l|}{} \\
\hline Officers & 7 & 17.1 \\
Agriculture & 19 & 46.3 \\
Students & 4 & 9.75 \\
Workers & 4 & 9.75 \\
Others & 7 & 17.1 \\
\hline Time interval to reach the PCC after snakebite \\
\hline <6 hours & 11 & 26.8 \\
6h-24 hours & 14 & 34.1 \\
24-72 hours & 15 & 36.6 \\
$>72$ hours & 1 & 2.4 \\
\hline \multicolumn{2}{|l|}{} \\
\hline Age & Mean & SD \\
Duration of hospitalization (days) & 5.63 & 3.29 \\
Glasgow point on admission & 15 & 0 \\
\hline Blood pressure & 118.68 & 16.00 \\
\hline Systolic & 74.39 & 10.50 \\
Diastolic & 97.83 & 2.07 \\
SpO ${ }_{2}$ on admission & 20.39 & 1.95 \\
Heart rate & 86.44 & 11.09 \\
Death & 0 & 0 \\
\hline
\end{tabular}

increased in $12(29.3 \%)$. Fibrinogen concentration was decreased in $61 \%$ and up to 18 (43.9\%) cases were severe decreased fibrinogen. Elevated D-dimer level was found in 36 (87.8\%). CT EXTEM prolonged, CT INTEM prolonged, CT FIBTEM prolonged, MCF FIBTEM decreased were $63.4 \%, 34.1 \%, 61 \%$ and $51.2 \%$, respectively (Table 3).

Table 4 shows the prolonged CT, CFT in INTEM, EXTEM, and FIBTEM. The clot amplitude at A5, A10, MCF in INTEM and EXTEM were all decreased.

After further adjustment for multiple correlates of coagulation disorder and time admission including age, gender, type of snake, the association between coagulation disorder and CT EXTEM prolonged, CT INTEM prolonged, CT FIBTEM prolonged remained significant (multivariable odds ratio $\mathrm{MOR}=5.81,95 \% \mathrm{CI}: 1.20-28.06$; $\mathrm{MOR}=9.32,95 \% \mathrm{CI}: 1.001-84.48 ; \mathrm{MOR}=5.55,95 \% \mathrm{CI}:$ $1.12-27.50$, respectively) (Table 5). 
Table 2 Characteristics of Patients without Coagulopathy, Patients with Simple DIC, and Patients with Overt DIC

\begin{tabular}{|c|c|c|c|c|}
\hline Variable & $\begin{array}{l}\text { Normal } \\
(D I C=0) \\
N=10(24.4)\end{array}$ & $\begin{array}{l}\text { Simple DIC } \\
\text { DIC I-4 } \\
N=I 7(4 I .5)\end{array}$ & $\begin{array}{l}\text { Overt DIC } \\
\text { DIC } \geq 5 \\
N=I 4(34 . I)\end{array}$ & $\mathbf{p}$ \\
\hline \multicolumn{5}{|l|}{ Snake species } \\
\hline Trimeresurus cornutus & $0(0.0)$ & I (5.9) & $0(0.0)$ & 0.004 \\
\hline Deinaglistrodon acutus & $0(0.0)$ & $0(0.0)$ & I (7.I) & \\
\hline Trimeresurus mucrosquamatus & $6(60.0)$ & $0(0.0)$ & I (7.I) & \\
\hline Trimeresurus albolabris & $2(20.0)$ & $10(58.8)$ & $10(71.4)$ & \\
\hline Undefined & $2(20.0)$ & $6(35.3)$ & $2(14.3)$ & \\
\hline \multicolumn{5}{|l|}{ Site of snakebite } \\
\hline Right arm & $5(50.0)$ & $2(11.8)$ & $3(2 \mid .4)$ & 0.229 \\
\hline Left arm & $0(0.0)$ & $4(23.5)$ & $4(28.6)$ & \\
\hline Right leg & $3(30.0)$ & $5(29.4)$ & $5(35.7)$ & \\
\hline Left leg & $2(20.0)$ & $6(35.3)$ & $2(14.3)$ & \\
\hline \multicolumn{5}{|l|}{ Clinical Symptoms } \\
\hline Local bleeding & $2(20.0)$ & $6(35.3)$ & $7(50.0)$ & 0.319 \\
\hline Swollen & $10(100.0)$ & $17(100.0)$ & $14(100.0)$ & - \\
\hline Fang marks & $10(100.0)$ & $17(100.0)$ & $14(100.0)$ & - \\
\hline Necrosis & $0(0.0)$ & I (5.9) & $\mathrm{I}(7.1)$ & 0.703 \\
\hline Blister & I $(10.0)$ & $2(11.8)$ & $3(2 \mid .4)$ & 0.67 \\
\hline Gastrointestinal hemorrhage & $0(0.0)$ & $0(0.0)$ & $3(2 \mid .4)$ & 0.044 \\
\hline \multicolumn{5}{|l|}{ Sex } \\
\hline Male & $6(60.0)$ & $10(58.8)$ & $9(64.3)$ & 0.950 \\
\hline \multirow[t]{2}{*}{ Female } & $4(40.0)$ & $7(4 \mid .2)$ & $5(35.7)$ & \\
\hline & Mean (SD) & Mean (SD) & Mean (SD) & \\
\hline $\mathbf{H b}$ & $139.50(21.43)$ & I35.94 (I5.83) & $121.79(26.99)$ & 0.212 \\
\hline WBC & $|2.4|(3.13)$ & $12.39(4.01)$ & $12.99(4.80)$ & 0.907 \\
\hline Creatine level & 78.5 (I5.69) & $80.18(20.76)$ & $82.86(29.35)$ & 0.896 \\
\hline Age & $38.2(10.73)$ & $43.0(15.65)$ & $41.36(16.56)$ & 0.725 \\
\hline Number of admission days & $5.2(2.66)$ & $5.29(2.22)$ & $6.36(4.63)$ & 0.609 \\
\hline Glassgow point at admission & $15.0(0.00)$ & $15.0(0.0)$ & $15.0(0.0)$ & - \\
\hline $\mathrm{SpO}_{2}$ on admission & $98.2(1.32)$ & $98.18(2.24)$ & $97.14(2.25)$ & 0.319 \\
\hline Respiratory rate & $19.9(1.52)$ & $20.06(1.60)$ & $21.14(2.45)$ & 0.203 \\
\hline Heart rate & $81.9(7.50)$ & $84.12(10.81)$ & 92.50 ( 11.52$)$ & 0.032 \\
\hline
\end{tabular}

\section{Discussion}

The study shows a high incidence of subclinical haematological abnormalities following bites by Viperidae. No patients died in our study although the patients with DIC accounted for $75.6 \%$. Delayed hospital admission was associated with coagulation disorders. Most of the patients were bitten by Trimeresurus albolabris (53.65\%) and Trimeresurus mucrosquamatus $(17.07 \%)$. Trimeresurus albolabris is widely distributed venomous species in Vietnam and lives mostly in the Northwest region. In some provinces of Vietnam (eg Lam Dong Province) it is the most frequent cause of snakebite., ${ }^{9,10}$ Trimeresurus 
Table 3 Characteristics of Coagulation Abnormalities of Patients with Snakebites at the Admission

\begin{tabular}{|c|c|c|}
\hline Characteristics & (n) & Percentage \\
\hline \multicolumn{3}{|l|}{ Platelet } \\
\hline Severe decreased $(<50)$ & 7 & 17.1 \\
\hline Mild decreased $(50-100)$ & 7 & 17.1 \\
\hline Normal $(>100)$ & 27 & 65.8 \\
\hline \multicolumn{3}{|l|}{ PT\% } \\
\hline Severe decreased $(<40 \%)$ & 8 & 19.5 \\
\hline Mild decreased (40-70\%) & 8 & 19.5 \\
\hline Normal ( $\geq 70 \%)$ & 25 & 61.0 \\
\hline \multicolumn{3}{|l|}{ APTTs } \\
\hline Prolong (>40s) & 7 & 17.1 \\
\hline Normal $(\leq 40 s)$ & 34 & 82.9 \\
\hline \multicolumn{3}{|l|}{ INR } \\
\hline Increased ( $(\geq 1.5)$ & 12 & 29.3 \\
\hline Normal (0.9-1.5) & 29 & 70.7 \\
\hline \multicolumn{3}{|l|}{ Fibrinogen } \\
\hline Severe decreased $(<1)$ & 18 & 43.9 \\
\hline Mild decreased (I-2) & 7 & 17.1 \\
\hline Normal $(>2)$ & 16 & 39.0 \\
\hline \multicolumn{3}{|l|}{ D-dimer (missing 2) } \\
\hline Severe increased $(\geq 5)$ & 21 & 51.2 \\
\hline Mild increased (2-5) & 15 & 36.6 \\
\hline Normal $(<1)$ & 3 & 7.3 \\
\hline \multicolumn{3}{|l|}{ ROTEM } \\
\hline CT EXTEM prolonged (>79s) & 26 & 63.4 \\
\hline CT INTEM prolonged (>240s) & 14 & 34.1 \\
\hline CT FIBTEM prolonged (>70s) & 25 & 61.0 \\
\hline MCF FIBTEM decreased $(<9 \mathrm{~mm})$ & 21 & 51.2 \\
\hline
\end{tabular}

mucrosquamatus has a wide geographic distribution. It causes a serious medical threat to people because of their overlapping habitats with areas of human activities, frequenting human dwellings in pursuit of prey, and inflicting severe envenomation. ${ }^{11}$ Trimeresurus mucrosquamatus were the venomous snakes of highest medical importance in Asia according to WHO. ${ }^{12}$

The study showed that coagulopathy resulting from viper envenoming is characterised by a low fibrinogen, platelet count and an elevated INR, D-dimer, and aPTT. The findings were similar to the published literature in Thailand, Sri Lanka. ${ }^{13-15}$ ROTEM abnormalities showed a hypocoagulation status (CT prolonged, MCF decreased) among Viper snakebite victims. Prolonged clotting time of the EXTEM and FIBTEM channel exhibits an exogenous and endogenous clotting pathway disorder. Coagulopathy after viper envenoming is a consequence of activation of the clotting pathway by procoagulant toxins. This will result in clotting factor consumption. The enzymatic toxins restricting with coagulation are anticoagulant proteases (protein C activator, factor IX inhibitors, factor $\mathrm{X}$ inhibitors, anticoagulant PLA2s) and pro-coagulant proteases (prothrombin activators, factor $\mathrm{X}$, thrombin-like enzymes and factor $\mathrm{V}$ activators). The venom elements acting on fibrinolysis are plasminogen activators and fibrinolytic enzymes. ${ }^{16,17}$ Viperid venoms are determined to act on platelets, and several venom components include high functional and structural similarities to unlike natural ligands of the platelet adhesion receptor, therefore affecting platelet functions due to different mechanisms. Venom elements contribute to venom-induced coagulopathy by depleting platelets and culminating in marked thrombocytopenia. $^{18,19}$ ISTH score were correlated to the ROTEM studies in Lund, Sweden, ${ }^{20}$ and Copenhagen, Denmark. $^{21}$

After adjusting for multiple correlates of coagulation disorder and time admission, our results showed that a prolonged arrival delay was associated with coagulation disorder (CT EXTEM prolonged, CT INTEM prolonged, CT FIBTEM prolonged). The Viperidae family comprises

Table 4 Characteristics of Disorders in ROTEM

\begin{tabular}{|l|l|l|l|l|l|l|}
\hline & CT (s) & CFT (s) & Alpha (độ) & A5 (mm) & Al0 (mm) & MCF (mm) \\
\hline EXTEM $(n=4 I)$ & $91(62 ; 267,5)$ & $165,5(99 ; 34 I)$ & $60,5(42 ; 72)$ & $28(16,5 ; 38,5)$ & $38(26 ; 48,5)$ & $48(29,5 ; 57,5)$ \\
\hline INTEM $(n=4 I)$ & $213(177,5 ; 358)$ & $165(92 ; 276,5)$ & $62(50 ; 7 I)$ & $28(17,5 ; 39,5)$ & $38(26,5 ; 49,5)$ & $48(36,5 ; 56)$ \\
\hline FIBTEM $(n=4 I)$ & $87(57,5 ; 2407)$ & - & - & $9(4 ; 12)$ & $10(4 ; 13)$ & $12(4 ; 14)$ \\
\hline APTEM $(n=4 I)$ & $8 I(6 I ; 26 I)$ & $156,5(95,5 ; 309)$ & $60(40 ; 70)$ & $27(12 ; 39,5)$ & $39(25 ; 49,5)$ & $49(36 ; 57)$ \\
\hline
\end{tabular}

Note: Values are presented as median (quartile range). 
Table 5 Relationship Between Coagulation Disorder and Duration from Snakebite Moment to Admission (>6 Hours)

\begin{tabular}{|l|l|l|l|l|}
\hline Characteristics & CT EXTEM Prolonged & CT INTEM Prolonged & CT FIBTEM Prolonged & MCF FIBTEM Decreased \\
\hline Model I & $5.37(1.15-24.97)^{*}$ & $9.93(1.01-85.48)^{*}$ & $5.03(1.06-23.80)^{*}$ & $0.24(0.05-1.12)$ \\
\hline Model 2 & $5.810(1.20-28.06)^{*}$ & $9.19(1.001-84.48)$ & $5.55(1.12-27.50)^{*}$ & $0.243(0.05-1.15)$ \\
\hline
\end{tabular}

Notes: ${ }^{*} \mathrm{p}<0.05$. Model I adjust to age, gender. Model 2 adjust to age, gender, type of snake.

a main group of venomous snakes and causes for the majority of mortality and morbidity worldwide compared to the others. ${ }^{19}$ After a bite from venomous snake, the coagulopathy could appear very quickly. This emphasizes the need to transport immediately the victim to the nearest medical facility. Previous study suggested first-aid measures such as incision, tourniquets and drainage, and indigenous herbal medicines are strongly discouraged. ${ }^{22}$

This study has several implications. Our study was among the first research providing insights about the coagulopathy after Viper snakebite in Vietnam. Coagulation process in the study was comprehensively evaluated through coagulation tests and thromboelastometry. Our research design has overcome the shortcomings with a predetermined protocol.

This study has several limitations to discuss. This study focused on patients at the center hospital. Thus, the patients included in this study may have more severe snakebites than people treated in local hospitals or in community settings. The number of patients was small. Larger number of patients are needed to get more specific conclusion.

\section{Conclusion}

In conclusion, our study indicates a hypocoagulation status in ROTEM, elevated INR, aPTT and D-dimer, decreased fibrinogen concentration and platelet count following envenoming by Viperidae. Delayed hospital admission was associated with coagulation disorders.

\section{Data Sharing Statement}

The datasets of this study are available from the corresponding author on reasonable request.

\section{Ethics Approval and Informed Consent}

The study was approved by the Ethics Committee of Bach Mai Hospital (reference number: 3377/QD-BM). We confirm that our study complied with the Declaration of Helsinki. Written informed consent was obtained from all participants. For patients who were not of legal age to sign on their own behalf, written informed consent was obtained from their guardians. The questionnaire did not include private information or any harm to participants. The result of research was used for the study purposes only.

\section{Acknowledgments}

We would like to express our deepest gratitude for the great support of Vietnam Poison Center, Bach Mai Hospital, Hanoi, Vietnam during research conducting.

\section{Author Contributions}

All authors made contributions to conception, design, conducting of data, or analysis and interpretation of data; participate in drafting the article or revising it critically for important intellectual content; agreed on the submitted journal and gave final approval of the version to be published.

\section{Funding}

This research received no external funding.

\section{Disclosure}

The authors reported no conflicts of interest for this work.

\section{References}

1. Casewell NR, Jackson TNW, Laustsen AH, et al. Causes and consequences of snake venom variation. Trends Pharmacol Sci. 2020;41 (8):570-581. doi:10.1016/j.tips.2020.05.006

2. Kasturiratne A, Wickremasinghe AR, de Silva N, et al. The global burden of snakebite: a literature analysis and modelling based on regional estimates of envenoming and deaths. PLoS Med. 2008;5 (11):e218. doi:10.1371/journal.pmed.0050218

3. Cheng AC, Winkel KD. Snakebite and antivenoms in the AsiaPacific: wokabaut wantaim, raka hebou ("walking together"). Med J Aust. 2001;175(11-12):648-651. doi:10.5694/j.1326-5377.2001. tb143762.x

4. Patra A, Mukherjee AK. Assessment of snakebite burdens, clinical features of envenomation, and strategies to improve snakebite management in Vietnam. Acta Trop. 2021;216:105833. doi:10.1016/j. actatropica.2021.105833 
5. Blessmann J, Nguyen TPN, Bui TPA, et al. Incidence of snakebites in 3 different geographic regions in Thua Thien Hue Province, Central Vietnam: green pit vipers and cobras cause the majority of bites. Toxicon. 2018;156:61-65. doi:10.1016/j.toxicon.2018.11.009

6. Mehta SR, Sashindran VK. Clinical features and management of snake bite. Med J Armed Forces India. 2002;58(3):247-249. doi:10.1016/S0377-1237(02)80140-X

7. Taylor FB Jr, Hoots TC, Wada H, Levi M, Levi M. Scientific subcommittee on Disseminated Intravascular Coagulation (DIC) of the International Society on Thrombosis and Haemostasis (ISTH). Towards definition, clinical and laboratory criteria, and a scoring system for disseminated intravascular coagulation. Thromb Haemost. 2001;86(5):1327-1330. doi:10.1055/s-0037-1616068

8. Crochemore T, Rodrigues PF, Rodrigues RDR, Guerra JCDC, Ferraz LJR, Corrêa TD. A new era of thromboelastometry. Einstein (Sao Paulo). 2017;15(3):380-385. doi:10.1590/s1679$45082017 \mathrm{md} 3130$

9. Thumtecho S, Tangtrongchitr T, Srisuma S, et al. Hematotoxic manifestations and management of green pit viper bites in Thailand. Ther Clin Risk Manag. 2020;16:695-704. doi:10.2147/ TCRM.S261303

10. Suen PY. Bamboo snake (trimeresurus albolabris) bite in Hong Kong and its prognostic factors for local complications with surgical intervention, coagulopathy and thrombocytopenia. Hong Kong J Emerg Med. 2017;24(2):79-84. doi:10.1177/102490791702400205

11. Hung DZ. Taiwan's venomous snakebite: epidemiological, evolution and geographic differences. Trans $R$ Soc Trop Med Hyg. 2004;98 (2):96-101. doi:10.1016/S0035-9203(03)00013-0

12. Chippaux JP. [Guidelines for the production, control and regulation of snake antivenom immunoglobulins]. Biol Aujourdhui. 2010;204 (1):87-91. French. doi:10.1051/jbio/2009043

13. Rojnuckarin P, Intragumtornchai T, Sattapiboon R, et al. The effects of green pit viper (Trimeresurus albolabris and Trimeresurus macrops) venom on the fibrinolytic system in human. Toxicon. 1999;37(5):743-755. doi:10.1016/S0041-0101(98)00214-1
14. Hutton RA, Looareesuwan S, Ho M, et al. Arboreal green pit vipers (genus Trimeresurus) of South-East Asia: bites by T. albolabris and T. macrops in Thailand and a review of the literature. Trans $R$ Soc Trop Med Hyg. 1990;84(6):866-874. doi:10.1016/0035-9203(90) 90111-Q

15. Isbister GK, Maduwage K, Scorgie FE, et al. Venom concentrations and clotting factor levels in a prospective cohort of russell's viper bites with coagulopathy. PLoS Negl Trop Dis. 2015;9(8):e0003968. doi:10.1371/journal.pntd.0003968

16. Du X-Y, Sim DS, Lee W-H, et al. Blood cells as targets of snake toxins. Blood Cells Mol Dis. 2006;36(3):414-421. doi:10.1016/j. bcmd.2006.03.001

17. Kini RM. Toxins in thrombosis and haemostasis: potential beyond imagination. J Thromb Haemost. 2011;9(Suppl 1):195-208. doi:10.1111/j.1538-7836.2011.04279.x

18. de Queiroz MR, de Sousa BB, da Cunha Pereira DF, et al. The role of platelets in hemostasis and the effects of snake venom toxins on platelet function. Toxicon. 2017;133:33-47. doi:10.1016/j. toxicon.2017.04.013

19. Matsui T, Hamako J, Titani K. Structure and function of snake venom proteins affecting platelet plug formation. Toxins (Basel). 2010;2 (1):10-23. doi:10.3390/toxins2010010

20. Kander T, Larsson A, Taune V, Schött U, Tynngård N. Assessment of haemostasis in disseminated intravascular coagulation by use of point-of-care assays and routine coagulation tests, in critically ill patients; a Prospective Observational Study. PLoS One. 2016;11(3): e0151202. doi:10.1371/journal.pone.0151202

21. Ostrowski SR, Windelov NA, Ibsen M, Haase N, Perner A, Johansson PI. Consecutive thrombelastography clot strength profiles in patients with severe sepsis and their association with 28-day mortality: a Prospective Study. J Crit Care. 2013;28(3):317e1-e11. doi:10.1016/j.jcrc.2012.09.003

22. Gold BS, Dart RC, Barish RA. Bites of venomous snakes. $N$ Engl $J$ Med. 2002;347(5):347-356. doi:10.1056/NEJMra013477
Journal of Multidisciplinary Healthcare

\section{Publish your work in this journal}

The Journal of Multidisciplinary Healthcare is an international, peerreviewed open-access journal that aims to represent and publish research in healthcare areas delivered by practitioners of different disciplines. This includes studies and reviews conducted by multidisciplinary teams as well as research which evaluates the results or conduct of such teams or healthcare processes in general. The journal covers a very wide range of areas and welcomes submissions from practitioners at all levels, from all over the world. The manuscript management system is completely online and includes a very quick and fair peer-review system. Visit http://www.dovepress.com/testimonials. php to read real quotes from published authors. 